Research Paper

\title{
Genetic Polymorphisms of Transcription Factor NRF2 and of its Host Gene Sulfiredoxin (SRXN1) are Associated with Cerebrovascular Disease in a Finnish Cohort, the TAMRISK Study
}

\author{
Tarja Kunnas, Kirsi Määttä, Seppo T Nikkari ${ }^{\bowtie}$ \\ Department of Medical Biochemistry, University of Tampere Medical School and Fimlab laboratories, Tampere, Finland. \\ $\square$ Corresponding author: Seppo Nikkari, MD, PhD, Department of Medical Biochemistry, University of Tampere Medical School, 33014 University of Tampere,
} Finland. Phone: +358 503969 639; E-mail: seppo.nikkari@uta.fi.

(C) Ivyspring International Publisher. Reproduction is permitted for personal, noncommercial use, provided that the article is in whole, unmodified, and properly cited. See http://ivyspring.com/terms for terms and conditions.

Received: 2015.12.30; Accepted: 2016.02.26; Published: 2016.04.10

\begin{abstract}
Oxidative stress is involved in the pathophysiology of many cardiovascular disorders, such as hypertension and atherosclerosis. NRF2 is the primary transcriptional regulator of several antioxidant genes, including that of sulfiredoxin (SRXN1). The association of genotypes of NRF2 and SRXN1 with cardiovascular conditions was studied in a Finnish cohort of 336 subjects with diagnosed hypertension and 480 normotensive controls from the Tampere adult population cardiovascular risk study (TAMRISK). Samples were genotyped for four SNPs (rs1962142, rs2706110, rs6721961 and rs6706649) in the NRF2 gene region and four SNPs (rs6053666, rs61 16929, rs2008022, rs6085283) in the SRXN1 gene region using Competitive Allele Specific PCR (KASP) technique. Cardiovascular diseases were followed up from 2005 to 2014 using the Finnish National Hospital Discharge Registry (HILMO). Four out of eight studied polymorphisms: rs6721961, rs1962142, rs2706110 of NRF2, and rs6053666 of SRXN1 were associated with cerebrovascular disease. NRF2 polymorphism rs6721961 showed association with hypertension. NRF2 and SRXN1 polymorphisms, previously thought to be associated with human disease, appear to be associated particularly with cerebrovascular disease.
\end{abstract}

Key words: NF-E2-Related Factor 2/genetics; Genetic Predisposition to Disease/genetics; Oxidoreductases Acting on Sulfur Group Donors/genetics; Cerebrovascular Disorders.

\section{Introduction}

The cellular defense response to oxidative stress includes induction of detoxifying- and antioxidant enzymes. Nuclear factor (erythroid-derived 2) -like 2, also known as NFE2L2 or NFR2, is a transcription factor, which binds to the antioxidant response elements (ARE) -bearing genes during oxidative stress, activating their transcription [1]. One of the antioxidant genes that NRF2 activates is sulfiredoxin (SRXN1), an enzyme that reactivates peroxiredoxins (Prxs), when they are inhibited by over oxidation [2]. Prxs are important peroxidases that play different roles in reducing hydrogen peroxide. They are involved in roles such as protecting DNA against mutation and suppressing tumor formation [3].

It is thought that NRF2 plays an important role in antioxidant defenses in cardiovascular diseases, such as atherosclerosis and hypertension [4]. On the other hand, there is evidence that NRF2 activity may also accelerate the pathogenesis of some cardiovascular diseases [5]. The precise conditions under which NRF2 acts to attenuate or stimulate cardiovascular disease processes are unclear [4]. Genetic polymorphisms of NRF2 and SRXN1 have been shown to be associated with breast cancer [6] and other human diseases [7], implicating that genetic mechanisms associated with reactive oxygen species and NRF2 pathway are involved in disease initiation and progression. In order to assess whether these polymorphisms are also associated with cardiovascular events, we examined putative 
functional SNPs, four single-nucleotide polymorphisms on NRF2 gene and four on SRXN1, in subjects with hypertension and in normotensive controls in a Finnish cohort of 50-year-old subjects followed up for 10 years

\section{Materials and Methods}

\section{Subjects}

TAMRISK study data was collected from periodic health examinations (PHE) done for a Finnish 50-year-old PHE cohort $(n=6000)$ [8]. Hypertensive subjects $(n=336)$ (diagnosed by a physician) and normotensive subjects $(n=480)$ of the same sex, similar smoking habits, and successful genotyping were chosen in the order of admission. The body mass index (BMI) was calculated from recorded height $(\mathrm{cm})$ and weight $(\mathrm{kg})$. Serum cholesterol (mmoles/l) was measured after an overnight fast by standard techniques. Basic evaluation in 2003 included an interview by a public health nurse using a structured questionnaire about diseases and health-related behaviour. Buccal swabs for DNA extraction, and a-permission form for the use of PHE information and national registry data were collected by mail separately from the physical examination during years 2006-2010. Using the patient's national identity code, data on hospitalizations including ICD-10 codes for discharge diagnoses were obtained from the Finnish obligatory National Hospital Discharge Registry (HILMO) maintained by the National Institute of Health and Welfare. Prevalence of hypertension (I10-I15), ischemic heart diseases (I20-I25), and incidence of cerebrovascular diseases (I60-I69) and transient cerebral ischemic attacks (TIA) (G45) were followed up from 2005 to 2014 until the subjects were on the average 60 years old. In follow-up of the genotyped subjects, there were 78 with ischemic heart disease, 15 who had a diagnosis of cerebrovascular disease and 8 with TIA; a total of 98 had diagnosis of any one of the above cardiovascular diseases. The Ethics Committees of the Tampere University Hospital and the City of Tampere approved the study.

\section{Genotyping}

DNA was extracted from buccal swabs using a commercial kit (Qiagen Inc., Valencia, Calif., USA). The samples were transferred into 96-well plates and genotyped for NRF2 (rs1962142, rs2706110, rs6721961 and rs6706649) and SRXN1 (rs6053666, rs6116929, rs2008022, rs6085283) polymorphisms at the KBioscience Institute (UK) using Competitive Allele Specific PCR (KASP) technique.

\section{Statistical analysis}

Chi-square test for categorical variables was applied for the comparison of genotype groups. Association analyses for cardiovascular events were done also by logistic regression with gender and BMI as cofactors. Analyses were carried out using SPSS 20.0 for Windows (SPSS Inc., Chicago, Illinois, USA).

\section{Results}

Two putative functional SNPs (rs1962142 and rs2706110) and two functional SNPs (rs6721961 and rs6706649) [9] were analyzed in the NRF2 gene region [10]. Four putative functional SNPs (rs6053666, rs6116929, rs2008022, rs6085283) were analyzed in the SRXN1 gene region [10]. The frequencies of all the studied polymorphisms satisfied Hardy-Weinberg proportions $(\mathrm{P}>0.05)$.

Clinical characteristics of case group of hypertensive subjects (336) and controls (480) at the age of 50 years have been previously described [8]. The statistical analyses of clinical characteristics between different genotype groups for the different polymorphisms are given in Table 1 . The analyses were also adjusted for BMI and gender with logistic regression.

The NRF2 rs6721961 (G>T) genotype TT was associated with cerebrovascular diseases. In logistic regression, subjects with genotype TT had an 8.8-fold risk compared with those with GG ( $p=0.009$, CI 1.725 - 44.937), although they had lower risk for hypertension $(p=0.009, \quad O R=0.133$, CI $0.029-0.610)$ compared with $\mathrm{G}$ allele carriers. However, subjects with genotype TG had a 1.4-fold risk for hypertension than those with GG ( $p=0.034, C I 1.028-2.058)$.

NRF2 rs1962142 (C>T) genotype TT was associated with an increase of cerebrovascular diseases. More specifically, subjects with genotype TT had a 14-fold risk compared with carriers of the CC genotype ( $p=0.002$, CI 2.653-76.961). NRF2 rs2706110 $(\mathrm{C}>\mathrm{T})$ was also associated with an increased risk of cerebrovascular diseases. Subjects with genotype TT had a 4.9-fold risk compared with those with CC $(p=0.017$, CI 1.337-18.227). However, NRF2 rs6706649 showed no such association.

SRXN1 rs6053666 (T>C) TT genotype was associated with increased risk of cerebrovascular diseases (including TIA). Subjects with genotype TT had a 6.5-fold risk compared with those with CC $(\mathrm{p}=0.071, \mathrm{CI} 0.850-50.507)$. SRXN1 $\operatorname{rs} 6116929(\mathrm{G}>\mathrm{A})$, SRXN1 rs6085283 (C>T) and SRXN1 rs2008022 (C>A) showed no association with cardiovascular disease. 
Table 1. Clinical characteristics of the study population stratified according to NRF2 and SRXN1 genotypes. P values $<0.05$ are in bold.

\begin{tabular}{|c|c|c|c|c|c|}
\hline & & & & $\mathbf{P}$ & $\mathbf{P}^{*}$ \\
\hline NRF2 rs6721961 & GG & TG & TT & & \\
\hline Frequency (n) & $0.706(577)$ & $0.273(222)$ & $0.021(17)$ & & \\
\hline Ischemic heart diseases (\%) & 9.5 & 9.9 & 5.9 & 0.862 & 0.978 \\
\hline Cerebrovascular diseases + TIA (\%) & 3.1 & 1.8 & 11.8 & 0.058 & 0.093 \\
\hline Cerebrovascular diseases (\%) & 1.6 & 1.8 & 11.8 & 0.009 & 0.032 \\
\hline Hypertension (\%) & 42.3 & 48.8 & 12.5 & 0.011 & 0.004 \\
\hline NRF2 rs1962142 & $\mathrm{CC}$ & TC & TT & & \\
\hline Frequency (n) & $0.748(603)$ & $0.206(190)$ & $0.016(13)$ & & \\
\hline Ischemic heart diseases (\%) & 9.6 & 9.5 & 7.7 & 0.967 & 0.983 \\
\hline Cerebrovascular diseases + TIA $(\%)$ & 2.8 & 2.6 & 15.4 & 0.029 & 0.062 \\
\hline Cerebrovascular diseases (\%) & 1.3 & 2.6 & 15.4 & 0.001 & 0.007 \\
\hline Hypertension (\%) & 43.7 & 44.8 & 16.7 & 0.161 & 0.101 \\
\hline NRF2 rs2706110 & $\mathrm{CC}$ & TC & TT & & \\
\hline Frequency (n) & $0.511(409)$ & $0.411(329)$ & $0.079(63)$ & & \\
\hline Ischemic heart diseases $(\%)$ & 10.5 & 8.2 & 12.7 & 0.409 & 0.309 \\
\hline Cerebrovascular diseases + TIA (\%) & 3.2 & 2.1 & 6.3 & 0.188 & 0.181 \\
\hline Cerebrovascular diseases $(\%)$ & 1.5 & 1.5 & 6.3 & 0.024 & 0.035 \\
\hline Hypertension (\%) & 45.1 & 43.0 & 36.2 & 0.433 & 0.600 \\
\hline NRF2 rs6706649 & $\mathrm{CC}$ & CT & TT & & \\
\hline Frequency (n) & $0.788(639)$ & $0.206(167)$ & $0.06(5)$ & & \\
\hline Ischemic heart diseases (\%) & 9.0 & 12.5 & 0.00 & 0.306 & 0.409 \\
\hline Cerebrovascular diseases + TIA (\%) & 2.8 & 3.6 & 0.0 & 0.808 & 0.976 \\
\hline Cerebrovascular diseases (\%) & 1.9 & 1.8 & 0.0 & 0.951 & 0.973 \\
\hline Hypertension (\%) & 42.8 & 46.0 & 60.0 & 0.583 & 0.888 \\
\hline SRXN1 rs6053666 & TT & TC & $\mathrm{CC}$ & & \\
\hline Frequency (n) & $0.378(304)$ & $0.465(377)$ & $0.157(126)$ & & \\
\hline Ischemic heart diseases $(\%)$ & 9.5 & 11.4 & 4.0 & 0.040 & 0.117 \\
\hline Cerebrovascular diseases + TIA (\%) & 4.9 & 2.1 & 0.8 & 0.029 & 0.040 \\
\hline Cerebrovascular diseases & 3.3 & 1.1 & 0.8 & 0.064 & 0.077 \\
\hline Hypertension (\%) & 44.4 & 42.7 & 43.0 & 0.903 & 0.888 \\
\hline SRXN1 rs6116929 & GG & GA & AA & & \\
\hline Frequency (n) & $0.277(218)$ & $0.478(376)$ & $0.244(192)$ & & \\
\hline Ischemic heart diseases $(\%)$ & 10.9 & 8.0 & 11.5 & 0.307 & 0.254 \\
\hline Cerebrovascular diseases + TIA (\%) & 0.9 & 3.2 & 4.7 & 0.069 & 0.119 \\
\hline Cerebrovascular diseases (\%) & 0.0 & 2.4 & 2.6 & 0.063 & 0.053 \\
\hline Hypertension (\%) & 39.9 & 46.5 & 41.8 & 0.262 & 0.505 \\
\hline SRXN1 rs6085283 & $\mathrm{CC}$ & TC & TT & & \\
\hline Frequency (n) & $0.352(285)$ & $0.473(383)$ & $0.175(142)$ & & \\
\hline Ischemic heart diseases (\%) & 8.8 & 10.4 & 9.9 & 0.771 & 0.832 \\
\hline Cerebrovascular diseases + TIA ( $\%)$ & 1.1 & 3.9 & 4.2 & 0.060 & 0.078 \\
\hline Cerebrovascular diseases (\%) & 0.4 & 2.9 & 2.1 & 0.056 & 0.123 \\
\hline Hypertension (\%) & 42.8 & 45.2 & 41.5 & 0.698 & 0.526 \\
\hline SRXN1 rs2008022 & $\mathrm{CC}$ & CA & AA & & \\
\hline Frequency (n) & $0.605(483)$ & $0.339(271)$ & $0.056(45)$ & & \\
\hline Ischemic heart diseases (\%) & 9.1 & 11.7 & 6.7 & 0.382 & 0.594 \\
\hline Cerebrovascular diseases + TIA (\%) & 3.5 & 2.2 & 2.2 & 0.569 & 0.583 \\
\hline Cerebrovascular diseases (\%) & 2.3 & 1.5 & 0.0 & 0.467 & 0.750 \\
\hline Hypertension (\%) & 44.1 & 43.3 & 42.2 & 0.962 & 0.879 \\
\hline
\end{tabular}

$\mathrm{P}^{*}$, adjusted for gender and BMI

\section{Discussion}

On the basis of a recent finding on the association of certain NFR2 and SRXN1 polymorphisms with breast cancer [6], we hypothesized that they might be associated with cardiovascular disease as well, since reactive oxygen species (ROS) play a major role in vascular inflammation and pathophysiology [7]. In experimental conditions adenoviral gene transfer of NFR2 has in fact been shown to effectively reduce oxidative stress and inhibit vascular inflammation [11]. On the other hand, also opposite results have been obtained [5].

The rs6721961 $(\mathrm{G}>\mathrm{T})$ variation in the NRF2 promoter has previously shown to be functional [12]. 
The $\mathrm{T}$ allele associates with a low extent of NRF2 protein expression, resulting in lower SRXN1 expression and increased risk of breast cancer [6]. The $\mathrm{T}$ allele has also been associated with thromboembolism in women [13], impaired vasodilator responses [14], elevated blood pressure [15] and adenocarcinoma [16]. Using an animal model, Muthusamy et al. showed that increased amount of ROS activates NFR2 also in the myocardial tissue [17]. Disruption of NRF2 may lead to increased production of ROS because of the lack of antioxidant gene transcription. This could finally lead to cardiovascular complications, including myocardial infarction. However, induction of this pathway by different factors in a dose-dependent manner remains unknown. In our study, the rare TT genotype associated with increased cerebrovascular disease, and somewhat in contrast to an earlier study, decreased prevalence of hypertension [15]. On the other hand, our results are in agreement with those previous results, since compared to genotype GG, subjects with genotype TG had more hypertension. The other SNP lying in the $5^{\prime}$ regulatory region of NRF2 (rs 6706649) showed no association with cardiovascular disease.

Although most of the antioxidant-related disease-associated NRF2 variants are in the promoter region of the gene, thus affecting NFR2 gene regulation, there is also evidence that intronic variant rs 1962142 is associated with increased risk of disease. In breast cancer, the NRF2 rs1962142 (C>T) T allele was associated with a low level of cytoplasmic NRF2 expression and lower SRXN1 expression [6], which could be interpreted as diminished antioxidant capacity leading to disease risk. In our study, TT genotype was associated with increased risk of cerebrovascular disease. The NRF2 rs2706110 (C>T) T allele was previously associated with an increased risk of breast cancer [6]. In our study, TT genotype was associated with increased cerebrovascular risk.

In experimental conditions, NFR2 has been shown to influence atherosclerosis through its effects on plasma lipoproteins and cholesterol transport that overshadow its antioxidant protection [5]. Unexpectedly, increased NFR2 expression in mice promoted atherosclerotic lesion formation most likely by a combination of systemic metabolic and local vascular effects [5]. We found that NRF2 rs6706649 $(\mathrm{C}>\mathrm{T})$ genotype CC was associated significantly with increased serum cholesterol values compared to the TC genotype $(p=0.01)$, but other associations of any of the studied polymorphisms with serum cholesterol values were not observed (data not shown). However, NRF2 rs6706649 showed no association with cardiovascular diseases, and it has previously been shown not to be associated with vasodilator responses [14].

The rs6053666 $(\mathrm{T}>\mathrm{C})$ is situated on the $3^{\prime}$ untranslated region of the SRXN1 gene and may participate in alternative splicing [6]. The $\mathrm{C}$ allele of this polymorphic site was previously associated with a decrease in breast cancer risk [6]. According to our results, rs6053666 C allele was associated with a decrease of cerebrovascular diseases (including TIA), and before adjustment for gender and BMI, also with a decreased prevalence of ischemic heart disease. This tentatively indicates that SRXN1 gene may play a role in cardiovascular diseases in addition to cancer. However, SRXN1 rs6116929 and rs6085283, previously associated with decreased breast cancer survival, did not associate with cardiovascular disease, although regulatory features have been observed for the regions where they reside [18]. The variation SRXN1 rs2008022 showed no association with cardiovascular diseases either.

Limitations of the study include that the study group was restricted to residents of a large city in Finland, and the subjects were from a restricted genetic pool (Finnish Caucasian), which poses a challenge to how broadly the findings can be applied. Moreover, there were only 23 subjects in the follow-up who had a diagnosis of cerebrovascular disease, and the findings need further verification.

In conclusion, a total of three NRF2 polymorphisms and one SRXN1 variation showed association with cerebrovascular risk. All of these polymorphisms have previously been shown to be associated with breast cancer. It is possible that patients with insufficient NRF2 levels may be susceptible to disease development in the cerebrovascular system.

\section{Competing Interests}

The authors have declared that no competing interest exists.

\section{References}

1. Moi P, Chan K, Asunis I, et al. Isolation of NF-E2-related factor 2 (Nrf2), a NF-E2-like basic leucine zipper transcriptional activator that binds to the tandem NF-E2/AP1 repeat of the beta-globin locus control region. Proc Natl Acad Sci U S A 1994; 91: 9926-30.

2. Jonsson TJ, Johnson LC, Lowther WT. Protein engineering of the quaternary sulfiredoxin.peroxiredoxin enzyme.substrate complex reveals the molecular basis for cysteine sulfinic acid phosphorylation. J Biol Chem 2009; 284: 33305-10.

3. Perkins A, Poole LB, Karplus PA. Tuning of peroxiredoxin catalysis for various physiological roles. Biochemistry 2014; 53: 7693-705.

4. Howden R. Nrf2 and cardiovascular defense. Oxid Med Cell Longev 2013; 2013: 104308.

5. Barajas B, Che N, Yin F, et al. NF-E2-related factor 2 promotes atherosclerosis by effects on plasma lipoproteins and cholesterol transport that overshadow antioxidant protection. Arterioscler Thromb Vasc Biol 2011; 31: 58-66.

6. Hartikainen JM, Tengstrom M, Kosma VM, et al. Genetic polymorphisms and protein expression of NRF2 and Sulfiredoxin predict survival outcomes in breast cancer. Cancer Res 2012; 72: 5537-46.

7. Cho HY, Marzec J, Kleeberger SR. Functional polymorphisms in Nrf2: implications for human disease. Free Radic Biol Med 2015; 88: 362-72. 
8. Maatta KM, Nikkari ST, Kunnas TA. Genetic variant coding for iron regulatory protein HFE contributes to hypertension, the TAMRISK study. Medicine (Baltimore) 2015; 94: e464.

9. von Otter M, Landgren S, Nilsson S, et al. Association of Nrf2-encoding NFE2L2 haplotypes with Parkinson's disease. BMC Med Genet 2010; 11: 36-2350-11-36.

10. Barrett JC, Fry B, Maller J, et al. Haploview: analysis and visualization of LD and haplotype maps. Bioinformatics 2005; 21: 263-65.

11. Levonen AL, Inkala M, Heikura $\mathrm{T}$, et al. Nrf2 gene transfer induces antioxidant enzymes and suppresses smooth muscle cell growth in vitro and reduces oxidative stress in rabbit aorta in vivo. Arterioscler Thromb Vasc Biol 2007; 27: 741-47.

12. Marzec JM, Christie JD, Reddy SP, et al. Functional polymorphisms in the transcription factor NRF2 in humans increase the risk of acute lung injury. FASEB J 2007; 21: 2237-46.

13. Bouligand J, Cabaret $\mathrm{O}$, Canonico $\mathrm{M}$, et al. Effect of NFE2L2 genetic polymorphism on the association between oral estrogen therapy and the risk of venous thromboembolism in postmenopausal women. Clin Pharmacol Ther 2011; 89: 60-4.

14. Marczak ED, Marzec J, Zeldin DC, et al. Polymorphisms in the transcription factor NRF2 and forearm vasodilator responses in humans. Pharmacogenet Genomics 2012; 22: 620-8.

15. Shimoyama Y, Mitsuda Y, Tsuruta Y, et al. Polymorphism of Nrf2, an antioxidative gene, is associated with blood pressure and cardiovascular mortality in hemodialysis patients. Int J Med Sci 2014; 11: 726-31.

16. Okano Y, Nezu U, Enokida Y, et al. SNP (-617C $>$ A) in ARE-like loci of the NRF2 gene: a new biomarker for prognosis of lung adenocarcinoma in Japanese non-smoking women. PLoS One 2013; 8: e73794.

17. Muthusamy VR, Kannan S, Sadhaasivam K, et al. Acute exercise stress activates Nrf2/ARE signaling and promotes antioxidant mechanisms in the myocardium. Free Radic Biol Med 2012; 52: 366-76.

18. Lee PH, Shatkay H. F-SNP: computationally predicted functional SNPs for disease association studies. Nucleic Acids Res 2008; 36: D820-4. 